\title{
The application analysis of adaptive filter based on polynomial AR model in GPS dynamic positioning
}

\author{
Xianglei Wang ${ }^{\mathrm{a}}$ and Guitao Fu \\ Beijing Satellite Navigation Center, BEIJING, 100094
}

\begin{abstract}
When modeling for colored noise, order number and the coefficient of determination of the model is a difficult problem, real-time computing applicability is very good, although but increased filtering calculation burden. Therefore based on the polynomial approximation theory, this paper proposes a polynomial AR model to colored noise modeling, model coefficient and the order number and the polynomial order, only speed up the computing speed of the filter. Through an example for a GPS cycle slip detection effectiveness of the proposed algorithm is verified.
\end{abstract}

Keywords: GPS dynamic; AR model; kalman filter; colored noise; adaptive filter.

\section{Introduction}

In GPS dynamic positioning, the observation equations for nonlinear equations, general application of various nonlinear filtering algorithm. Such as extended kalman filter, based on deterministic sampling no trace of the kalman filter (UKF), kalman filtering (CKF) and volume based on sequential monte carlo particle filter (PF). Because PF prone to particle impoverishment in dealing with problems, and the problem of different sampling strategy are also different, so it is difficult to find a general model of the particle filter. At present, the processing non-linear problem with more or amount of calculation is relatively simple EKF, UKF and CKF.

In GPS observations, the remaining ionospheric delay, tropospheric delay error and the influence of multipath effect and so on, combined with GPS receiver of the sampling rate is higher and higher, make GPS observation noise, often do not conform to the gaussian white noise assumption, but non gaussian colored noise sequence. But the EKF, UKF, CKF are based on the observation noise is gaussian white noise, if you don't consider the effect of non gaussian colored noise, not only can reduce the estimation precision, but also the estimate result of filtering through optimization, thus generate inconsistencies, so for non-gaussian colored noise processing appears more important.

There are many methods to deal with the colored noise, direct modeling the colored noise is a important method which using he additional function model to compensate the error caused by colored gaussian noise model. Based on this, this article using the AR model is proposed for colored noise modeling, model coefficient and the order number is related to the polynomial order only, this method can improve the computing speed of the filter, last, an example is given which accounts for the effectiveness of the method.

\footnotetext{
a Corresponding author : chxywxl@163.com
} 


\section{Processing method with color noise}

Kind of method to deal with the impact of colored noise is directly analyze the colored noise modeling, attached to the function model, is used to compensate the error caused by colored gaussian noise model.

The state space model of GPS dynamic positioning as follows:

$$
\begin{aligned}
& \mathbf{X}_{k}=\boldsymbol{\Phi}_{k, k-1} \mathbf{X}_{k-1}+\mathbf{W}_{k} \\
& \mathbf{L}_{\mathbf{k}}=h\left(\mathbf{X}_{\mathbf{k}}\right)+\mathbf{u}\left(t_{k}\right)+\mathbf{e}_{\mathbf{k}}
\end{aligned}
$$

In the above format, $\boldsymbol{X}_{k}$ is for $t_{k}$ the moment of state vector, $\boldsymbol{\Phi}_{k, k-1}$ is state transition matrix for the $m \times m_{\text {order, }} \boldsymbol{W}_{k}$ is $m$ order state noise. $h(\bullet)$ As nonlinear observation model function, $\boldsymbol{e}_{k}$ is $n$ order the observation noise, according to the observation noise of white noise, $u(t)$ is said of non-gaussian noise, $t_{k}$ is real or random variables.

Non-gaussian colored noise power spectrum on frequency and can be regarded as a linear system under white noise driven response, due to the time series analysis method is used more convenient, thus become the most commonly used modeling methods. Time series analysis method the smooth sequence of colored noise as each moment related sequence and each moment of a white noise, the moment of $t_{k}$ non-gaussian colored noise $u\left(t_{k}\right)$ is expressed as:

$$
u\left(t_{k}\right)=\left(\gamma_{1} u\left(t_{k-1}\right)+\gamma_{2} u\left(t_{k-2}\right)+\cdots+\gamma_{p} u\left(t_{k-p}\right)\right)+\left(\xi_{k}-\beta_{1} \xi_{k-1}-\beta_{2} \xi_{k-2}-\cdots-\xi_{q} W_{k-q}\right)
$$

Here $\gamma_{i}<1(i=1, \cdots, p)$ known as the regression parameters, $\beta_{i}<1(i=1, \cdots, q)$ referred to as the moving average parameters. First part generally referred to as autoregressive model (Auto Regressive, AR) model, in the second part is called sliding (Moving Average, MA) model. Formula (3) is collectively referred to as autoregressive moving average (ARMA) model. When modeling in colored noise is analyzed, compared with single AR and MA models, to achieve the same accuracy, the parameters of the ARMA model requires less, better performance (Kaveh, 1979; Cadzow, 1980). But the determination of ARMA model parameters in essence is a nonlinear optimization problem, very difficult. So when the actual data processing, often with high order AR model of equivalent or higher order MA model for colored noise modeling (Graupe et al., 1975; Li S P et al., 1989; deng self-reliance, etc., 2003; Kizilkaya and Kayran, 2006).

Related literature research, based on the MA model to modeling colored noise, can adapt to the common colored noise sequence, based on the MA model compensation filter algorithm is the key to the MA model parameter identification, the MA model order selection is a more difficult problem. The first-order AR model based on traditional method to deal with colored noise, containing a colored noise is observed value converted to the corresponding values that contain white noise, then application related theory of filter state estimation. Is presented here based on AR model compensation algorithm of polynomial approximation theory.

\section{Adaptive filter based on polynomial AR model of compensation}

\subsection{Theory of AR model of the colored noise based on the polynomial approximation}

The state space model of the colored noise is regarded as:

$$
\mathbf{X}_{k}=\boldsymbol{\Phi}_{k, k-1} \mathbf{X}_{k-1}+\mathbf{W}_{k}
$$




$$
\mathbf{L}_{k}=h\left(\mathbf{X}_{k}\right)+\mathbf{u}\left(t_{k}\right)+\mathbf{e}_{k}
$$

Here, $\mathbf{u}\left(t_{k}\right)$ for the observation noise of colored noise, the ARMA model can be used in modeling, the Word decomposition theorem shows that limited variance ARMA model can approximate model by high order AR model. Which $\mathbf{u}\left(t_{k}\right)$ can be expressed as:

$$
D\left(z^{-1}\right) u\left(t_{k}\right)=\xi_{k}
$$

Where $D\left(z^{-1}\right)$ is the lag operator polynomial in the unit, its expression is:

$$
D\left(z^{-1}\right)=1+D_{1} z^{-1}+D_{2} z^{-2}+\ldots+D_{n_{d}} z^{-n_{d}}
$$

$D_{1} \cdots D_{n_{d}}$ is the coefficient, which $n_{d}$ are in order.

Based on the polynomial approximation theory to obtain the optimal coefficient $D_{1} \cdots D_{n_{d}}$, according to the theory of Stone - Weierstrass, within the limited observation time, any continuous function of time $t \mathrm{t}$ high order polynomial uniform approximation are available (Stone, 1937194; Xu L $\mathrm{J}$ et al., 2004).

$$
u\left(t_{k}\right)=\sum_{l=0}^{L} \beta_{l} k^{l}
$$

$\beta_{l}(l=0, \cdots, L)$ Which is a polynomial coefficients, $L$ is the polynomial order.

$t_{k}$ moment $u\left(t_{k}\right)$ can be predicted by the past value $n_{d}$.

$$
u\left(t_{k}\right)=\sum_{i=1}^{n_{d}} D_{i} u\left(t_{k-i}\right)+\xi_{k}
$$

For $^{u\left(t_{k}\right)}$, first consider the valuation $\hat{u}\left(t_{k}\right)$ should be unbiased, i.e.

$$
\mathrm{E}\left(\hat{u}\left(t_{k}\right)\right)=\sum_{i=1}^{n_{d}} D_{i} u\left(t_{k-i}\right)=u\left(t_{k}\right)
$$

On type is equivalent to the following two linear equality constraint (Heinonen and Neuvo, 1988; Harju and Ovaska, 1999).

$$
\begin{gathered}
\sum_{i=1}^{n_{d}} D_{i}=1 \\
\sum_{i=1}^{n_{d}} D_{i} i^{l}=0, l=1, \cdots, L-1
\end{gathered}
$$

Second consider valuation $\hat{u}\left(t_{k}\right)$ should be the best, with a minimum mean square error, the objective function can be constructed as follows:

$$
\mathrm{E}\left\{\left[\hat{u}\left(t_{k}\right)-u\left(t_{k}\right)\right]^{2}\right\}=\mathrm{E}\left\{\left[\sum_{i=1}^{n_{d}} D_{i} \xi_{k-i}\right]^{2}\right\}=\sum_{i=1}^{n_{d}} D_{i}^{2} \sigma_{\xi_{k-t}}^{2}=\min
$$


The type is equivalent to:

$$
\sum_{i=1}^{n_{d}} D_{i}^{2}=\min
$$

For the zero order, first order and second order and third order polynomial, the optimal coefficient $D_{1} \cdots D_{n_{d}}$ is (Heinonen and Neuvo, 1988) :

$$
\begin{gathered}
D_{i}=\frac{1}{n_{d}}, \quad L=0, i=1, \cdots, n_{d} \\
D_{i}=\frac{4 n_{d}-6 i+2}{n_{d}{ }^{2}-n_{d}}, \quad L=1, i=1, \cdots, n_{d} \\
D_{i}=\frac{9 n_{d}{ }^{2}+(9-36 i) n_{d}+30 i^{2}-18 i+6}{n_{d}{ }^{3}-3 n_{d}{ }^{2}+2 n_{d}}, \quad L=2, i=1, \cdots, n_{d} \\
D_{i}=\frac{16 n_{d}{ }^{3}+(24-120 i) n_{d}{ }^{2}+\left(240 i^{2}-120 i+56\right) n_{d}-140 i^{3}+120 i^{2}-100 i+24}{n_{d}{ }^{2}-6 n_{d}{ }^{3}+11 n_{d}{ }^{2}-6 n_{d}}, \quad L=3, i=1, \cdots, n_{d}
\end{gathered}
$$

It is can be seen from the above expression, AR model coefficient and the polynomial order number L, only order of AR model $n_{d}$, and has nothing to do with polynomial coefficients $\beta_{l}$, obviously, $n_{d} \geq L+1$ is a necessary condition, in the actual data processing, in order to reduce the computation burden, $n_{d}=L+1$ can be used. Figure 1 shows the zero order, first order and second order and third order polynomial, when order of AR model coefficient $n_{d}=10$. This also means that the AR model is used to model conforms to the polynomial form of non gaussian colored noise, except the polynomial order of thought, don't need other any priori information. AR model coefficient can be calculated in advance, very suitable for the need of real-time dynamic positioning. For high sampling rate of the observation value, in a short window, a second order polynomial is used to approximate colored noise is enough.

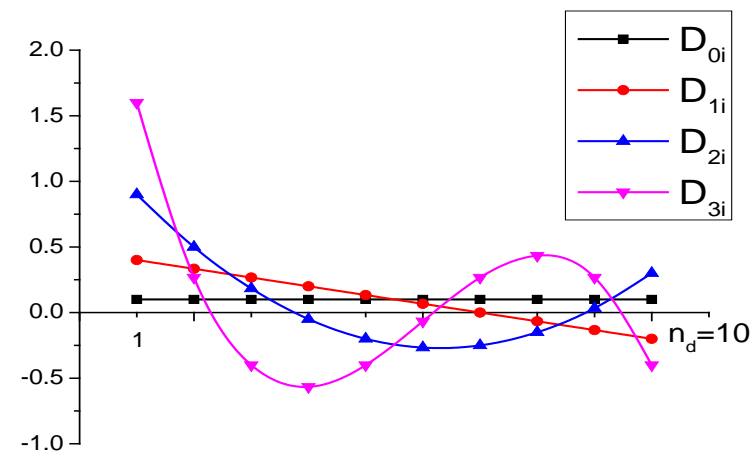

Figure 1. AR model coefficient polynomials 


\subsection{Polynomial approximation theory based on AR non-gaussian colored noise compensation filter}

According to the result of the previous section, the state space model based on AR model approximation of colored noise as follows:

$$
\begin{aligned}
& \mathbf{X}_{k}=\boldsymbol{\Phi}_{k, k-1} \mathbf{X}_{k-1}+\mathbf{W}_{k} \\
& \mathbf{L}_{k}=h\left(\mathbf{X}_{k}\right)+\mathbf{u}\left(t_{k}\right)+\mathbf{e}_{k} \\
& \mathbf{u}\left(t_{k}\right)=F_{u} \mathbf{u}\left(t_{k-1}\right)+\xi_{k}
\end{aligned}
$$

Here the third order AR model is used to approximate color noise, $F_{u}=\left[\begin{array}{lll}D_{1} & D_{2} & D_{3}\end{array}\right]$ as the AR model coefficient, by type (16) is computed. $\mathbf{W}_{k}, \mathbf{e}_{k}, \xi_{k}$ is gaussian white noise, and unrelated, the corresponding covariance matrix is $\Sigma_{\mathbf{W}_{k}}, \quad \sum_{\mathbf{e}_{k}}, \quad \Sigma_{\xi_{k}}$.

The above model for two phase deviation compensation filter, can look at is to omit by a deviation value without deviation of filter and a filter composed of two sub filter (Hsieh, and Chen, 1999).

No deviation filter (ignore the deviation value $\mathbf{u}\left(t_{k}\right)$, i.e. $\mathbf{u}\left(t_{k}\right)=0$,

Function model into:

$$
\begin{gathered}
\tilde{\mathbf{X}}_{k}=\boldsymbol{\Phi}_{k, k-1} \tilde{\mathbf{X}}_{k-1}+\mathbf{W}_{k} \\
\mathbf{L}_{k}=\mathbf{A}_{k} \tilde{\mathbf{X}}_{k}+\mathbf{e}_{k}
\end{gathered}
$$

Filter formula is:

$$
\begin{gathered}
\overline{\tilde{\mathbf{X}}}_{k}=\boldsymbol{\Phi}_{k, k-1} \hat{\tilde{\mathbf{X}}}_{k-1} \\
\boldsymbol{\Sigma}_{\overline{\tilde{\mathbf{X}}}_{k}}=\boldsymbol{\Phi}_{k, k-1} \boldsymbol{\Sigma}_{\overline{\tilde{\mathbf{X}}}_{k-1}} \boldsymbol{\Phi}_{k, k-1}^{T}+\boldsymbol{\Sigma}_{\mathbf{W}_{k}} \\
\tilde{\mathbf{K}}_{k}=\boldsymbol{\Sigma}_{\overline{\tilde{\mathbf{X}}}_{k}} \mathbf{H}_{k}^{T}\left(\mathbf{H}_{k} \boldsymbol{\Sigma}_{\tilde{\tilde{\mathbf{X}}}_{k}} \mathbf{H}_{k}^{T}+\boldsymbol{\Sigma}_{\mathbf{e}_{k}}\right)^{-1} \\
\hat{\tilde{\mathbf{X}}}_{k}=\overline{\tilde{\mathbf{X}}}_{k}+\tilde{\mathbf{K}}_{k}\left(\mathbf{L}_{k}-\mathbf{A}_{k} \overline{\tilde{\mathbf{X}}}_{k}\right) \\
\boldsymbol{\Sigma}_{\hat{\mathbf{x}}_{k}}=\left(\mathbf{I}-\tilde{\mathbf{K}}_{k} \mathbf{A}_{k}\right) \boldsymbol{\Sigma}_{\overline{\mathbf{X}}_{\mathbf{k}}} \\
\mathbf{H}_{\mathbf{k}}=\left.\frac{\partial \mathbf{h}\left(\mathbf{X}_{\mathbf{k}}\right)}{\partial \mathbf{X}_{k}}\right|_{\mathbf{X}_{\mathbf{k}}=\overline{\mathbf{X}}_{\mathbf{k}}}
\end{gathered}
$$

Deviation filter formula: 


$$
\begin{gathered}
\overline{\mathbf{u}}\left(t_{k}\right)=F_{u} \hat{\mathbf{u}}\left(t_{k-1: k-3}\right) \\
\bar{\Sigma}_{\overline{\mathbf{u}}\left(t_{k}\right)}=F_{u} \operatorname{diag}\left[\Sigma_{\hat{\mathbf{u}}\left(t_{k-1}\right)}, \boldsymbol{\Sigma}_{\hat{\mathbf{u}}\left(t_{k-2}\right)}, \boldsymbol{\Sigma}_{\hat{\mathbf{u}}\left(t_{k-3}\right)}\right] F_{u}^{T}+\Sigma_{\xi_{k}} \\
\hat{\mathbf{u}}\left(t_{k}\right)=\overline{\mathbf{u}}\left(t_{k}\right)+\mathbf{K}_{k}^{u}\left(\mathbf{L}_{k}-\mathbf{H}_{k} \tilde{\mathbf{X}}_{k}-\overline{\mathbf{u}}\left(t_{k}\right)\right) \\
\mathbf{K}_{k}^{u}=\bar{\Sigma}_{\overline{\mathbf{u}}\left(t_{k}\right)} \mathbf{S}_{k}^{T}\left[\mathbf{S}_{k} \overline{\mathbf{\Sigma}}_{\overline{\mathbf{u}}\left(t_{k}\right)} \mathbf{S}_{k}^{T}+\mathbf{H}_{k} \boldsymbol{\Sigma}_{\overline{\mathbf{x}}_{k}} \mathbf{H}_{k}^{T}+\boldsymbol{\Sigma}_{e_{k}}\right]^{-1} \\
\boldsymbol{\Sigma}_{\hat{\mathbf{u}}\left(t_{k}\right)}=\left(\mathbf{I}-\mathbf{K}_{k}^{u} \mathbf{S}_{k}\right) \overline{\boldsymbol{\Sigma}}_{\overline{\mathbf{u}}\left(t_{k}\right)}
\end{gathered}
$$

Here $\mathbf{S}_{k}=\mathbf{H}_{k} \mathbf{U}_{k}, \mathbf{U}_{k}=\boldsymbol{\Phi}_{k, k-1} \mathbf{N}_{k-1}, \mathbf{N}_{k}=\mathbf{U}_{k}-\tilde{\mathbf{K}}_{k} \mathbf{S}_{k}$

Eventually filter valuations state vector and covariance matrix of the:

$$
\begin{aligned}
& \hat{\mathbf{X}}_{k}=\hat{\tilde{\mathbf{X}}}_{k}+\mathbf{N}_{k} \hat{\mathbf{u}}\left(t_{k}\right) \\
& \boldsymbol{\Sigma}_{\dot{\mathbf{x}}_{k}}=\boldsymbol{\Sigma}_{\hat{\mathbf{x}}_{\mathbf{k}}}+\mathbf{N}_{k} \boldsymbol{\Sigma}_{\hat{\mathbf{u}}\left(t_{k}\right)} \mathbf{N}_{k}^{T}
\end{aligned}
$$

\section{Calculation and analysis}

Here by using AR model compensation based on polynomial approximation theory of adaptive filter algorithm to solve the problem of GPS cycle slip detection. The existing GPS cycle slip detection methods are polynomial fitting (preparation, 1997), the finite difference method (Zhou Zhongmo Yi Jiejun, Julie chi chow, 2004), the phase pseudorange group legal (shou-xin zhang, 1996), kalman filtering method (Jia Peizhang, even big wu, 2001), etc. These methods have their own characteristics, but also has its limitations: finite difference method is mainly used for detecting cycle slip, because of the difference method in the amplification of cycle slip also magnifies the noise; Polynomial fitting method to detect cycle slip, to ensure that at least the first four epoch carrier phase observation cycle slip, and then use them to detect and repair the fifth epoch carrier phase observations of cycle slip, the other two methods in the dynamic case cannot detect small cycle; Phase pseudorange group legal problem of cycle slip detecting ability depends on the amount of pseudorange observation precision, due to low precision GPS pseudorange observation in machine, so for small cycle slip detection ability is poor; While traditional kalman filtering method in when the observation noise is colored noise cannot effectively detect and repair cycle, even makes the filtering divergence.

Selection of satellite is PRN9, intercepting L1 observations of 600 epoch experiment. From 1 to 400 of them around the epoch observation environment better, epoch covered $400 \sim 600$, the observation environment starts to degrade can verify this section of the algorithm in weak strong observation of colored noise and color noise conditions for cycle slip detecting and repairing ability. 100 epoch in L1 observations with 1 week week, add 2 week at 300 epoch jump, jump at 440 epoch with 1 week, add 2 week at 550 epoch. First difference between epoch, to get a time series as a filter for observation, the aim is to make smooth carrier phase observation noise sequence.

Cycle slip detection USES two solutions:

Method1: standard Kalman filter (KF);

Method 2: AR model based on polynomial Kalman filtering (ARKF) compensation.

1) cycle slip detection and determined: the filter can get the carrier phase observations of prediction error sequence $\left\{\varepsilon_{k}\right\}$, according to former $\mathrm{k}-1$ epoch error value $\left\{\varepsilon_{k}\right\}$ can be obtained 


$$
\hat{\sigma}_{k-1}=\sqrt{\frac{\varepsilon_{1}^{2}+\varepsilon_{2}^{2}+\cdots+\varepsilon_{k-1}^{2}}{k-1}}
$$

For the first $\mathrm{k} \varepsilon_{k}$, if

$$
\varepsilon_{k}>h \hat{\sigma}_{k-1}
$$

Thinks that contain cycle, known as the door threshold coefficient. According to a large number of practical experience, take $h=2$. $K$ is called the data window wide, take is $K=8$.

When detected the moment $t_{k}$ have a cycle slip, can happen under type is used to estimate the size of the cycle.

$$
\text { Cycleslips }=\operatorname{round}\left(\varepsilon_{k}-h \sigma_{k-1}\right)
$$

Cycleslips is Valuation of cycle slip type, for the moment. Round Round.

Result of specific cycle slip detection and determine are shown in table 1, table 2.

Table 1. cycle slip detecting and repairing(method 1$)$

\begin{tabular}{c|cccc}
\hline \multirow{2}{*}{ epoch } & $\begin{array}{c}\text { L1 predict } \\
\varepsilon_{k}\end{array}$ & MSE $\sigma_{k-1} /$ cycle & Y/N & \multicolumn{2}{c}{$\begin{array}{c}\text { Cycle detect } \\
\text { error } c_{k}\end{array}$} \\
\hline 100 & 0.897 & 0.018 & $\mathrm{Y}$ & 1 \\
300 & -2.01 & 0.03 & $\mathrm{Y}$ & -2 \\
440 & 1.54 & 0.78 & $\mathrm{~N}$ & 0 \\
550 & 2.2658 & 0.79 & $\mathrm{Y}$ & 1 \\
\hline
\end{tabular}

Table 2. cycle slip detecting and repairing(method 2)

\begin{tabular}{c|cccc}
\hline \multirow{2}{*}{ epoch } & $\begin{array}{c}\text { L1 predict } \\
\varepsilon_{k}\end{array}$ & MSE $\sigma_{k-1} /$ cycle & Y/N & \multicolumn{2}{c}{$\begin{array}{c}\text { Cycle detect } \\
\text { result }\end{array}$} \\
\hline 100 & 1.03 & 0.016 & $\mathrm{Y}$ & 1 \\
300 & -2.02 & 0.023 & $\mathrm{Y}$ & -2 \\
440 & 1.67 & 0.34 & $\mathrm{Y}$ & 1 \\
550 & 2.223 & 0.35 & $\mathrm{Y}$ & 2 \\
\hline
\end{tabular}

From the calculation results can draw the following conclusion:

(1) when the GPS observation condition is good, the observation data quality is high, the standard Kalman filter and compensation based on polynomial AR model of Kalman filtering for cycle slip detecting and repairing effect is very good, for a week or so small can accurate cycle slip detection and repair.

(2) when the observation condition, carrier phase observation noise as gaussian white noise, not the standard Kalman filtering for cycle slip detecting and repairing effect also begins to be bad, can be seen from table 1, 440 epoch week cycle, has been submerged in the residual sequence, cannot detect. And 550 epoch of 2 week week, while it is possible to detect, but already can't repair.

(3) can be seen from table 2, the AR model based on polynomial compensation Kalman filter can effectively reduce the influence of colored noise, and still be able to detect and repair cycle.

\section{Conclusion}

Using polynomial AR model can effectively to modeling of colored noise, by modeling can effectively reduce the influence for the observation noise, not only such, still can use polynomial Kalman filtering 
of AR model compensation to small cycle slip detecting and repairing, in the use of AR model based on polynomial compensation Kalman filtering, the polynomial order generally can't more than three order, can undertake trial determined according to the specific problem, can also adopt the method of integrated model.

\section{Acknowledgement}

This work is supported by Research on Algorithm of Gnss Multistations Networking for Atomic Clocks Realtime Comparison (Grant NO. 11403112).

\section{References}

1. Gordon,N.J, Salmond,D.J, Smith,A.F.M., Novel Approach to Nonlinear/ Non-Gaussian Bayesian State Estimation. IEE Proceedings, Pt. F. 140, pp. 107-113, April(1993).

2. Julier, S. J., and Uhlmann, J. K., A new extension of the Kalman filter to nonlinear systems. Proceedings of SPIE 3, 182-193(1997).

3. Jwo, D.-J. and Lai, S.-Y. (2009), Navigation integration using the fuzzy strong tracking unscented Kalman filter.The Journal of Navigation, 62(2), pp.303-322.

4. K.Kramer and S.Stubberud, An adaptive Gaussian sum approach for maneuver tracking, Proc. of the IEEE Aerospace Conference, Big Sky, MT, 2083-2091(2005).

5. Kitagawa G., Monte Carlo filter and smoother for non-Gaussian nonlinear state space models. J.Comput.Graph.Statist. 5(1):1-25(1996).

6. DENG Zi-li. Kalman Filtering and Wiener filtering[M]. Haerbin, Harbin industrial university press, 2001.

7. DENG Zi-li, MA Jian-wei, DU Hong-yue. ARMA model parameter estimation of two least squares algorithm[J]. Science technology and engineering, 3(1):1-3, (2003).

8. HUANG Xian-yuan, SUI Li-fen, FAN Peng-pai. Random model series of coloured observation noise and its compensation method[J]. Journal of wuhan university. Information sciences,33(6):644-646,(2008).

9. FANG Chong-zhi, XIAO De-yun. Process to identify[M]. BEIJING:Tsinghua university press, 1988:173-175.

10. Ikoma, N., Ichimura, N., Higuchi, T., and Maeda, H., Particle filter based method for maneuvering target tracking. In Proceedings of the IEEE International Workshop of Intelligent Signal Processing, Budapest, Hungary,3-8(2010).

11. I. Arasaratnam and S. Haykin, Cubature Kalman Filters. IEEE Transactions on Automatic Control, vol. 54, no. 6, pp. 1254-1269(2009).

12. I. Arasaratnam, S. Haykin, and R. J. Elliott, Discrete time nonlinear filtering algorithms using gauss hermite quadrature. Proceedings of the IEEE, 95:953-977(2007). 